\title{
Gut microbiota-dependent trimethylamine-N-oxide (TMAO) Shows a U-shaped Association With Mortality But Not With Recurrent Venous Thromboembolism
}

Martin F. Reiner, ${ }^{*} \dagger^{1}$ Daniel Müller, $\ddagger^{1}$ Sara Gobbato, ${ }^{*} †$ Odile Stalder, § Andreas Limacher, § Nicole R. Bonetti, ${ }^{*}$ Lisa Pasterk, ${ }^{*}+$ Marie Méan, If Nicolas Rodondi, ${ }^{*}+\dagger$ Drahomir Aujesky, ${ }^{* \star}$ Anne

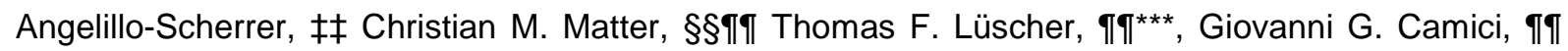
Arnold vonEckardstein, $\ddagger$ Jürg $\mathrm{H}$. Beer, * ${ }^{*}$

${ }^{1}$ equal contribution

${ }^{*}$ Center for Molecular Cardiology, Laboratory for Platelet Research, University of Zurich, Schlieren, Switzerland. †Department of Internal Medicine, Cantonal Hospital of Baden, Baden, Switzerland. ‡Institute of Clinical Chemistry, University Hospital Zurich, University of Zurich, Zurich, Switzerland. SCTU Bern and Institute of Social and Preventive Medicine (ISPM), University of Bern, Bern, Switzerland. đDivision of Internal Medicine, Lausanne University Hospital, Lausanne, Switzerland. **Department of General Internal Medicine, Inselspital Bern University Hospital, University of Bern, Bern, Switzerland. ††Institute of Primary Health Care (BIHAM), University of Bern, Bern, Switzerland. $\ddagger \ddagger S e r v i c e$ and Central Laboratory of Hematology, Lausanne University Hospital, Lausanne, Switzerland. §§Department of Cardiology, University Heart Center, University Hospital Zurich, Zurich, Switzerland. ॠTCenter for Molecular Cardiology, University of Zurich, Schlieren, Switzerland. ${ }^{* \star}$ Royal Brompton and Harefield Hospital Trust and Imperial College, London, United Kingdom

\section{Running head: TMAO and recurrent VTE}

Text word count: 3'302

\section{Abstract word count: 243}

Prof. Dr. med. Jürg Hans Beer, M.D., F.A.C.P.

Head of the Department of Medicine

Cantonal Hospital Baden

Im Ergel 1, CH-5404 Baden, Switzerland and Laboratory for Platelet Research, Center for Molecular Cardiology University of Zurich, Switzerland Phone: (++41) 5648625 02; Fax: (++41) 5648625 09; E-mail: hansjuerg.beer@ksb. 


\begin{abstract}
Introduction: Gut microbiota-dependent trimethylamine-N-oxide (TMAO) correlates with arterial thrombotic events including myocardial infarction and stroke, and mortality. However, the association of TMAO with recurrent venous thromboembolism (VTE) and mortality remains unknown.
\end{abstract}

Methods: TMAO plasma levels were assessed by high performance liquid chromatography in 859 patients aged $\geq 65$ years with acute VTE and categorized into low $(<2.28 \mu \mathrm{mol} / \mathrm{L})$, medium $(2.28-6.57 \mu \mathrm{mol} / \mathrm{L})$, and high levels $(>6.57 \mu \mathrm{mol} / \mathrm{L})$ based on the $25^{\text {th }}$ and $75^{\text {th }}$ percentile. Associations of TMAO with recurrent VTE, major or non-major bleeding, and mortality were investigated.

Results: During a mean follow-up of 28 months, 106 patients developed recurrent VTE, 259 had major or non-major bleeding events, and 179 patients died. The risk of recurrent VTE did not differ significantly between patients with low, medium (adjusted subhazard ratio [SHR], 1.38; $95 \%$ confidence interval [Cl], 0.81 to 2.36; $p=0.232$ ) and high TMAO levels (SHR, 1.44; $95 \% \mathrm{Cl}, 0.80$ to $2.58, \mathrm{p}=0.221)$. Compared with low TMAO levels, the adjusted hazard ratio [HR] for mortality was $0.68(95 \% \mathrm{Cl}, 0.47$ to $0.98, \mathrm{p}=0.039)$ in patients with medium TMAO levels and $1.02(95 \% \mathrm{Cl}, 0.68$ to $1.52, \mathrm{p}=0.922)$ in patients with high TMAO levels. Fractional polynomial Cox-regression confirmed a $U$-shaped association (adjusted $p=0.045$ ), with the lowest mortality risk in patients with TMAO around $4 \mu \mathrm{mol} / \mathrm{L}$. TMAO was not associated with major or non-major bleeding.

Conclusion: TMAO showed a U-shaped association with mortality in elderly patients with acute VTE and was not associated with recurrent VTE and major or non-major bleeding. 
Key words: Bleeding, deep vein thrombosis, pulmonary embolism, recurrent venous thromboembolism, trimethylamine-N-oxide 


\section{Abbreviations}

$\mathrm{Cl}$

HR

IQ

DVT

PE

SHR

TMAO

VTE confidence interval

hazard ratio

interquartile

deep vein thrombosis

pulmonary embolism

subhazard ratio

trimethylamine- $\mathrm{N}$-oxide

venous thromboembolism 


\section{Introduction}

Venous thromboembolism (VTE) consists of deep vein thrombosis (DVT) and pulmonary embolism (PE); its incidence ranges between 1.4 to 2.2 per 1,000 person-years and increases exponentially with age. [1] Anticoagulation is considered standard therapy to reduce recurrent VTE; however, a residual thrombotic risk remains and importantly, bleeding risk increases substantially. [2] In the absence of long-term anticoagulation, up to $30 \%$ of patients suffer recurrent VTE in 10 years. [1] Due to the high incidence of recurrent VTE and post-thrombotic complications, [1] substantial VTE-related healthcare costs [3] and bleeding complications, [2] further strategies are needed to reduce the burden of VTE.

Trimethylamine-N-oxide (TMAO), a gut microbiota-dependent metabolite of dietary phosphatidylcholine [4] and L-carnitine, [5] has been recently shown to increase the risk of arterial thrombotic events, such as myocardial infarction and stroke and consequently, overall mortality. [6, 7] Potential mechanisms underlying these observations include the propagation of atherosclerosis through activated monocytes/macrophages [4] as well as increased platelet activation and subsequent thrombotic arterial occlusion. [8] TMAO increases cholesterol uptake in macrophages leading to foam cell formation and thereby augments atherosclerotic lesion size converting arterial walls thrombogenic. [4] Further, it increases platelet aggregation and arterial thrombosis in rodents as well as human platelet adhesion and aggregation. [8] Both monocytes and platelets play a crucial role in the development of VTE. [9] During the initiation of venous thrombosis, monocytes and neutrophils are the dominant leukocytes adhering to venous vessel walls; likewise, platelets adhere to the endothelium and enhance leukocyte recruitment and neutrophil extracellular trap formation, [9] which is considered a crucial scaffold for venous thrombogenesis and in return activates platelet aggregation. [10-12] In line with this, platelet inhibition next to anticoagulation is recommended to prevent recurrent VTE. [2]

To date, the role of TMAO in venous thrombosis remains unknown. We therefore examined the association of TMAO plasma levels with recurrent VTE, major or non-major bleeding, and mortality in a prospective multicenter cohort study of 859 elderly patients with acute VTE. 


\section{Methods}

\section{Study population}

The current study is a sub-study of the Swiss Cohort of Elderly Patients with Venous Thromboembolism (SWITCO65+; ClinicalTrials.gov Identifier: NCT00973596), a prospective multicenter cohort study of elderly patients with acute VTE. [13] Patients were recruited from September 2009 to March $2012(n=1003)$. Inclusion criteria were age above 65 years and symptomatic, objectively confirmed VTE (proximal and/or distal DVT and/or PE). [13] Symptomatic, objectively confirmed DVT was defined as proximal and/or distal DVT detected by an objective imaging exam (ultrasonography, spiral computed tomography or magnetic resonance imaging venography) in a patient with symptoms. [13] Symptomatic, objectively confirmed PE was defined as a PE detected by spiral computed tomography, a highprobability ventilation-perfusion scintigraphy, pulmonary angiography or in the presence of objectively confirmed, symptomatic or asymptomatic proximal DVT in a patient with symptoms. [13] Exclusion criteria were inability to provide informed consent, conditions incompatible with follow-up, language barriers, thrombosis at a different site than lower limb, catheter-related thrombosis, or previous enrolment in the cohort. [13] After exclusion of 860 patients (no consent, $n=398$; inability to provide consent, $n=285$; follow-up not possible, $n$ $=192$; other site than lower $\operatorname{limb}, \mathrm{n}=21$; catheter-related thrombosis, $\mathrm{n}=7$; language barriers, $n=51$; multiple reasons for exclusion may apply), 1003 patients were enrolled and 144 patients were excluded from the current analysis (patients denying use of data, $n=8$; no biobank samples, $n=129$; no TMAO measurement, $n=7$ ); finally, TMAO was analyzed in 859 patients. The study received ethical approval from every participated center and eligible patients provided informed consent.

\section{Baseline data collection and follow-up}

At baseline, demographic information, comorbidities, laboratory findings, VTE-related treatment before and after the event, and concomitant antiplatelet therapy were collected using standardized data collection forms. Follow-up contact included one telephone interview 
and two face-to-face evaluations during the first year followed by semi-annual contacts and periodic reviews of the patient's hospital chart. Patients were followed-up for 28 months on average.

\section{Blood sampling and determination of TMAO}

At baseline, heparin-anticoagulated blood was drawn after minimal venostasis; aliquots of samples were cryovialed in $3 \mathrm{ml}$ polypropylene tubes and temporarily stored at $-80^{\circ} \mathrm{C}$ before they were transported to and stored at the SWITCO65+ biobank at the Central Laboratory of Hematology of Lausanne University hospital, Switzerland.

For the determination of TMAO, plasma samples were shipped to the Institute of Clinical Chemistry, University Hospital Zurich (Zurich, Switzerland). Levels of TMAO were quantified by liquid chromatography-mass spectrometry, similar as previously described. [14] Briefly, $400 \mu \mathrm{L}$ of an internal standard mixture containing TMAO-d9, at $1 \mu \mathrm{mol} / \mathrm{L}$ was added, vortexed and centrifuged $\left(11^{\prime} 700 \mathrm{~g}, 10 \mathrm{~min}, 4^{\circ} \mathrm{C}\right)$ and supernatant was analyzed. Separation was achieved on an Accucore HILIC column $(50 \times 2.1 \mathrm{~mm}, 2.6 \mu \mathrm{m}$ particle size, Thermo Fisher Scientific, Reinach, Switzerland) under acidic conditions. As mass spectrometer, a QTrap 6500 hybrid instrument (Sciex) was used, that acquired chromatograms in positive electrospray ionization multi-reaction monitoring mode. The following transitions were used: $76.1 \rightarrow 59.1$ (quantifier), $76.1 \rightarrow 42.1$ (qualifier 1), $76.1 \rightarrow 56.2$ (qualifier 2) for TMAO and $85.1 \rightarrow 68.1$ for TMAO-d9. The modified method was validated and showed a good comparability to the original method described in [14]. All researchers involved in TMAO determination were blinded to patient characteristics and outcomes.

\section{End points}

The endpoints of the current study were recurrent VTE, major or non-major bleeding, and mortality. Recurrent VTE was defined as new fatal or new non-fatal PE, or new DVT (proximal and/or distal). [13] Diagnosis of recurrent DVT was performed by abnormal results on ultrasonography; recurrent PE was diagnosed by computed tomography, angiography, or 
ventilation-perfusion lung scan; a new proximal DVT, associated with new PE symptom(s) was also considered as recurrent PE. [13] Major bleeding was defined as a fatal bleeding, symptomatic bleeding in a critical organ (intracranial, intraspinal, intraocular, retroperitoneal, intraarticular, pericardial, or intramuscular with compartment syndrome), a reduction of hemoglobin $\geq 20 \mathrm{~g} / \mathrm{L}$ or transfusion $\geq 2$ units of packed red blood cells. [13] Non-major bleeding was defined as bleedings that did not fulfill the criteria for major bleeding but required medical attention. [13]

\section{Statistical analysis}

TMAO plasma levels were categorized into low, medium, and high levels based on the $25^{\text {th }}$ and $75^{\text {th }}$ percentile (low, $<2.28 \mu \mathrm{mol} / \mathrm{L}$; medium, $2.28-6.57 \mu \mathrm{mol} / \mathrm{L} ;$ high, $>6.57 \mu \mathrm{mol} / \mathrm{L}$ ). We compared baseline characteristics of patients with different levels of plasma concentration of TMAO using the chi-squared test and the non-parametric Kruskal-Wallis test as appropriate. We estimated the cumulative incidence of a first VTE recurrence, a first major or non-major bleeding, and death during the first three years using the Kaplan-Meier method and compared survivor functions between groups by the log-rank test. Associations between plasma concentration of TMAO levels and the time to a first VTE recurrence and a first major or non-major bleeding were assessed by competing risk regression accounting for death as a competing event, according to the method of Fine and Gray [15]. The method yields subhazard ratios (SHR) with corresponding 95\% confidence intervals (Cls) and p-values for the failure event of primary interest. For death, an ordinary Cox-regression was calculated. We adjusted the models for risk factors that had previously been shown to be associated with VTE recurrence, major or non-major bleeding, or mortality. VTE recurrence was adjusted for age, gender, overt PE, prior VTE, provoked index VTE and periods of anticoagulation. Mortality was adjusted for age, gender, overt PE, active cancer, immobilization during the last 3 months, chronic or acute heart failure, chronic lung disease and periods of anticoagulation. Bleeding was adjusted for age, active cancer, history of major bleeding, antiplatelet therapy, overt PE, and periods of anticoagulation. We verified 
proportional-hazards assumptions using Schoenfeld residuals and time interactions. There was no significant interaction between time and TMAO levels, i.e. the effect of TMAO on outcomes did not change over time. Because a fractional polynomial Cox-regression suggested a U-shaped relationship between TMAO and death, we further investigated a quadratic relationship between log-transformed TMAO and death using a Cox model with a linear and quadratic term. Number of events and incidence rates per 100 person-years were displayed at 36 months for the total cohort and different TMAO levels. All analyses were done using Stata 14 (Stata Corporation, College Station, Texas). STROBE guidelines have been the basis for reporting our results. [16] 


\section{Results}

\section{Study population}

The mean age was 75 years, and $45 \%$ patients were female. The mean TMAO level was $6.51 \mu \mathrm{mol} / \mathrm{L}$. Patients' baseline characteristics were largely comparable across TMAO categories. However, patients with high TMAO levels were slightly older, had less provoked index VTEs (defined as presence of $\geq 1$ of the following factors: major surgery, oestrogen therapy, or immobilization during the last 3 months), had reduced glomerular filtration rates and were more likely to be hypertensive (Table 1). Mean follow-up was 28 months.

\section{Recurrent VTE}

During follow-up, 106 patients developed recurrent VTE. Patients with medium and high levels of TMAO were more likely to develop recurrent VTE after 3 years (low, $11.0 \%$; medium, $15.8 \%$; high, $16.1 \% ; p=0.393$ ) (Figure 1). After adjustment, medium TMAO levels were associated with a $38 \%$ (adjusted SHR, 1.38; 95\% Cl, 0.81 to $2.36 ; \mathrm{p}=0.232$ ) and high levels with a $44 \%$ (adjusted SHR, $1.44 ; 95 \% \mathrm{Cl}, 0.80$ to $2.58 ; \mathrm{p}=0.221$ ) higher risk of developing recurrent VTE compared to low levels of TMAO; however, these observations did not reach statistical significance (Table 2).

\section{Mortality}

Death occurred in 179 patients during follow-up. The cumulative incidence of mortality differed significantly between TMAO levels (low, $22.5 \%$; medium, $17.4 \%$; high, $27.7 \%$; p = 0.030) (Figure 2). After adjustment, the risk of mortality was significantly lower in patients with medium, as compared to patients with low levels of TMAO (adjusted hazard ratio [HR], $0.68 ; 95 \% \mathrm{Cl}, 0.47$ to $0.98 ; \mathrm{p}=0.039$ ) (Table 2). On the other hand, patients with high TMAO levels had a comparable risk to patients with low TMAO levels for dying (adjusted HR, 1.02; $95 \% \mathrm{Cl}, 0.68$ to $1.52 ; \mathrm{p}=0.922$ ) (Table 2) suggesting a U-shaped relationship between TMAO and mortality, which was confirmed in a fractional polynomial Cox-regression (Figure 
3). In a model with a linear and quadratic term, we found a significant quadratic relationship between log-transformed TMAO and death (adjusted joint $p=0.045$ ) (Table 3 ). The lowest number of events was observed in patients with TMAO around $4 \mu \mathrm{mol} / \mathrm{L}$ (Figure 3 ). At 3 years, a total of 179 deaths were observed: 60 deaths (33.5\%) were attributable to cancer, $33(18.4 \%)$ to PE (7 [3.9 \%] PE related, 26 [14.5\%] possibly PE related), 16 (8.9 \%) to sepsis, $14(7.8 \%)$ to infection, $12(6.7 \%)$ to left ventricular failure, $11(6.2 \%)$ to bleeding, 6 (3.4 \%) to pulmonary causes other than PE, $3(1.7 \%)$ to acute coronary syndrome, 2 (1.1\%) to stroke, $1(0.6 \%)$ to suicide, $5(2.8 \%)$ to others, and $16(8.9 \%)$ to unknown causes.

\section{Major or non-major bleeding}

The endpoint major or non-major bleeding occurred in 259 patients during follow-up. The cumulative incidence of major or non-major bleeding was comparable among different levels of TMAO (low, $36.0 \%$; medium, $33.5 \%$; high, $36.3 \%, p=0.817$ ) (Figure 4). Likewise, after adjustment, the risk of major or non-major bleeding in patients with medium (adjusted SHR, 0.96; $95 \% \mathrm{Cl}, 0.72$ to $1.28 ; \mathrm{p}=0.775$ ) and high TMAO levels (adjusted SHR, $0.94 ; 95 \% \mathrm{Cl}$, 0.67 to $1.32 ; p=0.732$ ) were comparable to low levels of TMAO (Table 2).

\section{Subgroup analysis for mortality}

In general, patients with PE are at higher risk of death, compared to patients with DVT. [1] In order to investigate whether the observed U-shaped association of TMAO and mortality was different in patients with PE compared to patients with DVT only, we performed a subgroup analysis. In our study, we found a comparable mortality between the two groups (Supplemental Table 1). The U-shaped association (i.e. lower risk in patients with medium as compared to low and high TMAO levels) was more pronounced in patients with PE than in patients with DVT only. However, a test for interaction between VTE type (PE versus DVT only) and TMAO levels was not significant.

We also performed a subgroup analysis in patients with and without cancer, because cancer was the major contributor to mortality in the current study. The U-shaped association was 
more pronounced in patients with cancer. However, a test for interaction between cancer and TMAO levels was not significant. Unfortunately, we cannot draw strong conclusions from these subgroup analyses, because $95 \%$ confidence intervals were rather wide and the power to detect differences between groups rather small. 


\section{Discussion}

In the current prospective multicenter cohort study we examined the association of gut microbiota-dependent TMAO with recurrent VTE, major or non-major bleeding, and mortality in patients at 65 years of age or above with acute VTE. We found that patients with medium and high TMAO levels had a $38 \%$ and $44 \%$ higher risk of developing recurrent VTE, respectively, compared with low levels of TMAO; however, these findings did not reach statistical significance. Interestingly, the risk of death was only reduced in patients with medium, but not in patients with high TMAO levels, as compared with low TMAO levels and we found a significant U-shaped association between log-transformed TMAO and mortality. More precisely, patients with TMAO plasma levels around $4 \mu \mathrm{mol} / \mathrm{L}$ had the lowest risk of death. Lastly, TMAO levels were not associated with major or non-major bleeding.

Previous studies have investigated the associations between TMAO plasma levels and arterial thrombotic events including myocardial infarction and stroke as well as mortality in patients with previous myocardial infarction or in patients at high risk of cardiovascular events. $[4,6,7,14,17,18]$ Most $[4,6,7]$, but not all of them $[14,17,18]$ found high TMAO levels to be associated with an increased risk of atherothrombotic diseases. Potential pathophysiological mechanisms include the propagation of atherosclerosis by increased activation of monocytes/macrophages [4] and augmented arterial thrombosis mediated by increased platelet activation and aggregation through TMAO. [8] Although both monocytes and platelets are crucial for VTE development [9], we did not find a significant association between TMAO and recurrent VTE. In this study, median TMAO levels in the medium and high groups were $3.9 \mu \mathrm{mol} / \mathrm{L}$ and $10.6 \mu \mathrm{mol} / \mathrm{L}$, respectively. Previous experimental studies showed that 10 to $31 \mu \mathrm{mol} / \mathrm{L}$ of TMAO were required in order to affect human platelet aggregation ex vivo and that arterial thrombosis was increased in mice with TMAO plasma levels of $100 \mu \mathrm{mol} / \mathrm{L}$; [8] further, monocyte/macrophage activation and atherosclerosis was augmented in mice with TMAO plasma levels between 20 and $70 \mu \mathrm{mol} / \mathrm{L}$. [4] Thus, one explanation for the missing association between TMAO and recurrent VTE might be that TMAO in the current study was slightly too low to affect venous thrombogenesis. In addition, 
the studied population is of advanced age, which per se is associated with elevated levels of coagulation factors and inflammatory mediators; [19] in addition, VTE itself triggers inflammation and coagulation [20] and these mechanisms may have played a more relevant role for the development of VTE than the effects of TMAO on monocytes and platelets.

The observation of a U-shaped association between log-transformed TMAO and mortality is novel. In contrast to our findings, previous longitudinal studies in patients with acute coronary syndrome, stable coronary heart disease, acute heart failure, chronic kidney disease or diabetes have reported linear associations between TMAO and mortality. [6, 7, 21-23] In addition, a recent meta-analysis in patients with coronary artery disease and patients at risk of cardio- and cerebrovascular disease also showed a positive direct and non-linear association of TMAO with mortality. [24] In this study we found that at baseline, patients with high TMAO levels were slightly older, had lower glomerular filtration rates and were more likely to be hypertensive. Mortality was corrected for age and the main causes of death were attributable to cancer and pulmonary embolism, which are not affected by hypertension and kidney function. TMAO is a gut microbiota-dependent metabolite and antibiotic treatment decreases TMAO levels substantially. [4, 6] Therefore, concomitant infectious diseases and the use of antibiotic therapy could explain the higher incidence of death in patients with low TMAO. Plasma levels of TMAO depend on the intake of fish as well as phosphatidylcholine and L-carnitine present in dairy products, eggs and red meat; therefore, malnutrition may be associated with both decreased TMAO levels and increased risk of mortality. [25] Body mass indices were comparable between the groups, which excludes underweight in patients with low TMAO; however, a normal body mass index does not exclude malnutrition in elderly patients. [26]

Interestingly, TMAO levels in the present study were slightly higher than in previous Central European populations [14, 27-29] possibly due the advanced age of the current population and/or the high prevalence of comorbidities such as chronic kidney disease, glucose intolerance, and inflammation, conditions that have previously been shown to be associated with higher plasma levels of TMAO. [14, 29] 
Strengths of our study include the large sample size. Further, TMAO measurements were performed in a standardized and reproducible manner. [14] The current study has several limitations. First, patients were recruited in Switzerland and were 65 years or above; thus, the findings are not applicable to the general population. Further, the high but non-significant SHR and the rather wide $95 \%$ confidence interval for recurrent VTE $(1.48(95 \% \mathrm{Cl} 0.88$ to 2.49) and 1.56 (95\% $\mathrm{Cl} 0.87$ to 2.77$)$ for medium and high TMAO levels, respectively) indicates a limited power to detect significant differences. The study is observational and only detects associations but not causality. Next, despite extensive adjustment, we cannot entirely exclude the possibility that the observed associations may be due to residual confounding or chance. Lastly, TMAO exhibits relevant intra-individual variation of roughly $45 \%$ over a 1year period [28] and single measurements may not be representative for long-term follow-up. The association of TMAO with venous thrombosis remained unknown so far. In the current study we found a trend for an increased risk of recurrent VTE in the elderly, which, however, was not statistically significant. Whether a larger sample size may had reached significant results remains to be determined. Further, the association of TMAO with recurrent VTE in younger patients or in patients with primary rather than recurrent VTE should be investigated in future studies. A U-shaped relationship of TMAO with mortality has not been reported earlier and may be specific to patients with VTE or to an elderly population due to comorbidities and/or nutritional habits. Whether pharmacological changes of TMAO affect recurrent VTE, bleeding or mortality remains to be determined in randomized controlled intervention trials.

In conclusion, TMAO showed a significant U-shaped association with mortality in elderly patients with acute VTE. The lowest number of events was observed in patients with TMAO around $4 \mu \mathrm{mol} / \mathrm{L}$. Contrary to arterial thrombosis, TMAO was not associated with recurrent VTE and TMAO did not correlate with major or non-major bleeding. 


\section{Addendum}

MFR, DM, OS, AL, MM, NR, DA, AAS, TFL, AvE and JHB designed the study. MFR, DM, SG, OS, AL, MM, NR, DA, AAS, and JHB performed measurements and analysis. MFR, DM, SG, OS, AL, NRB, LP, MM, NR, DA, AAS, CMM, TFL, GGC, AvE and JHB interpreted results. MFR, DM, SG, OS, AL, NRB, LP, MM, NR, DA, AAS, CMM, TFL, GGC, AvE and JHB wrote the manuscript. All authors critically read and revised the manuscript and approved the final version to be published.

\section{Acknowledgements}

The Swiss National Science Foundation supported the SWITCO65+ study (grant no. 33CSCO-122659/139470) and the current sub-study (grant no. 163339 to JHB). Furthermore, we received financial support from the Foundation Kardio, Baden, Switzerland. We thank all participating centers and collaborators of SWITCO65+.

\section{Disclosure of Conflicts of Interest}

The authors have nothing to disclose. 


\section{References}

1 Benjamin EJ, Blaha MJ, Chiuve SE, Cushman M, Das SR, Deo R, de Ferranti SD, Floyd J, Fornage M, Gillespie C, Isasi CR, Jimenez MC, Jordan LC, Judd SE, Lackland D, Lichtman JH, Lisabeth L, Liu S, Longenecker CT, Mackey $\mathrm{RH}$, et al. Heart Disease and Stroke Statistics-2017 Update: A Report From the American Heart Association. Circulation. 2017; 135: e146-e603.

2 Kearon C, Akl EA, Ornelas J, Blaivas A, Jimenez D, Bounameaux H, Huisman M, King CS, Morris TA, Sood N, Stevens SM, Vintch JR, Wells P, Woller SC, Moores L. Antithrombotic Therapy for VTE Disease: CHEST Guideline and Expert Panel Report. Chest. 2016; 149: 315-52.

3 Ruppert A, Lees M, Steinle T. Clinical burden of venous thromboembolism. Current medical research and opinion. 2010; 26: 2465-73.

4 Wang Z, Klipfell E, Bennett BJ, Koeth R, Levison BS, Dugar B, Feldstein AE, Britt EB, Fu X, Chung YM, Wu Y, Schauer P, Smith JD, Allayee H, Tang WH, DiDonato JA, Lusis AJ, Hazen SL. Gut flora metabolism of phosphatidylcholine promotes cardiovascular disease. Nature. 2011; 472: 57-63.

5 Koeth RA, Wang Z, Levison BS, Buffa JA, Org E, Sheehy BT, Britt EB, Fu X, Wu Y, Li L, Smith JD, DiDonato JA, Chen J, Li H, Wu GD, Lewis JD, Warrier M, Brown JM, Krauss $\mathrm{RM}$, Tang $\mathrm{WH}$, et al. Intestinal microbiota metabolism of L-carnitine, a nutrient in red meat, promotes atherosclerosis. Nature medicine. 2013; 19: 576-85.

6 Tang WH, Wang Z, Levison BS, Koeth RA, Britt EB, Fu X, Wu Y, Hazen SL. Intestinal microbial metabolism of phosphatidylcholine and cardiovascular risk. The New England journal of medicine. 2013; 368: 1575-84.

7 Wang Z, Tang WH, Buffa JA, Fu X, Britt EB, Koeth RA, Levison BS, Fan Y, Wu Y, Hazen SL. Prognostic value of choline and betaine depends on intestinal microbiotagenerated metabolite trimethylamine-N-oxide. European heart journal. 2014; 35: 904-10. 
8 Zhu W, Gregory JC, Org E, Buffa JA, Gupta N, Wang Z, Li L, Fu X, Wu Y, Mehrabian M, Sartor RB, McIntyre TM, Silverstein RL, Tang WH, DiDonato JA, Brown JM, Lusis AJ, Hazen SL. Gut Microbial Metabolite TMAO Enhances Platelet Hyperreactivity and Thrombosis Risk. Cell. 2016; 165: 111-24.

9 von Bruhl ML, Stark K, Steinhart A, Chandraratne S, Konrad I, Lorenz M, Khandoga A, Tirniceriu A, Coletti R, Kollnberger M, Byrne RA, Laitinen I, Walch A, Brill A, Pfeiler S, Manukyan D, Braun S, Lange P, Riegger J, Ware J, et al. Monocytes, neutrophils, and platelets cooperate to initiate and propagate venous thrombosis in mice in vivo. The Journal of experimental medicine. 2012; 209: 819-35.

10 Martinod K, Demers M, Fuchs TA, Wong SL, Brill A, Gallant M, Hu J, Wang Y, Wagner DD. Neutrophil histone modification by peptidylarginine deiminase 4 is critical for deep vein thrombosis in mice. Proceedings of the National Academy of Sciences of the United States of America. 2013; 110: 8674-9.

11 Brill A, Fuchs TA, Savchenko AS, Thomas GM, Martinod K, De Meyer SF, Bhandari AA, Wagner DD. Neutrophil extracellular traps promote deep vein thrombosis in mice. Journal of thrombosis and haemostasis : JTH. 2012; 10: 136-44.

12 Fuchs TA, Brill A, Duerschmied D, Schatzberg D, Monestier M, Myers DD, Jr., Wrobleski SK, Wakefield TW, Hartwig JH, Wagner DD. Extracellular DNA traps promote thrombosis. Proceedings of the National Academy of Sciences of the United States of America. 2010; 107: 15880-5.

13 Mean M, Righini M, Jaeger K, Beer HJ, Frauchiger B, Osterwalder J, Kucher N, Lammle B, Cornuz J, Angelillo-Scherrer A, Rodondi N, Limacher A, Trelle S, Matter CM, Husmann M, Banyai M, Aschwanden M, Egloff M, Mazzolai L, Hugli O, et al. The Swiss cohort of elderly patients with venous thromboembolism (SWITCO65+): rationale and methodology. Journal of thrombosis and thrombolysis. 2013; 36: 475-83.

14 Mueller DM, Allenspach M, Othman A, Saely $\mathrm{CH}$, Muendlein A, Vonbank A, Drexel H, von Eckardstein A. Plasma levels of trimethylamine-N-oxide are confounded by impaired kidney function and poor metabolic control. Atherosclerosis. 2015; 243: 638-44. 
15 Fine JP, Gray RJ. A proportional hazards model for the subdistribution of a competing risk. J Am Stat Assoc. 1999; 94: 496-509.

16 von Elm E, Altman DG, Egger M, Pocock SJ, Gotzsche PC, Vandenbroucke JP, Initiative S. Strengthening the Reporting of Observational Studies in Epidemiology (STROBE) statement: guidelines for reporting observational studies. BMJ. 2007; 335: 806-8. 17 Kaysen GA, Johansen KL, Chertow GM, Dalrymple LS, Kornak J, Grimes B, Dwyer T, Chassy AW, Fiehn O. Associations of Trimethylamine N-Oxide With Nutritional and Inflammatory Biomarkers and Cardiovascular Outcomes in Patients New to Dialysis. Journal of renal nutrition : the official journal of the Council on Renal Nutrition of the National Kidney Foundation. 2015; 25: 351-6.

18 Suzuki T, Heaney LM, Bhandari SS, Jones DJ, Ng LL. Trimethylamine N-oxide and prognosis in acute heart failure. Heart. 2016; 102: 841-8.

19 Rumley A, Emberson JR, Wannamethee SG, Lennon L, Whincup PH, Lowe GD. Effects of older age on fibrin D-dimer, C-reactive protein, and other hemostatic and inflammatory variables in men aged 60-79 years. Journal of thrombosis and haemostasis : JTH. 2006; 4: 982-7.

20 Bucek RA, Reiter M, Quehenberger P, Minar E. C-reactive protein in the diagnosis of deep vein thrombosis. British journal of haematology. 2002; 119: 385-9.

21 Tang WH, Wang Z, Fan Y, Levison B, Hazen JE, Donahue LM, Wu Y, Hazen SL. Prognostic value of elevated levels of intestinal microbe-generated metabolite trimethylamine-N-oxide in patients with heart failure: refining the gut hypothesis. Journal of the American College of Cardiology. 2014; 64: 1908-14.

22 Tang WH, Wang Z, Kennedy DJ, Wu Y, Buffa JA, Agatisa-Boyle B, Li XS, Levison BS, Hazen SL. Gut microbiota-dependent trimethylamine N-oxide (TMAO) pathway contributes to both development of renal insufficiency and mortality risk in chronic kidney disease. Circulation research. 2015; 116: 448-55. 
23 Tang WH, Wang Z, Li XS, Fan Y, Li DS, Wu Y, Hazen SL. Increased Trimethylamine N-Oxide Portends High Mortality Risk Independent of Glycemic Control in Patients with Type 2 Diabetes Mellitus. Clinical chemistry. 2017; 63: 297-306.

24 Schiattarella GG, Sannino A, Toscano E, Giugliano G, Gargiulo G, Franzone A, Trimarco B, Esposito G, Perrino C. Gut microbe-generated metabolite trimethylamine-Noxide as cardiovascular risk biomarker: a systematic review and dose-response metaanalysis. European heart journal. 2017; 38: 2948-56.

25 O'Shea E, Trawley S, Manning E, Barrett A, Browne V, Timmons S. Malnutrition in Hospitalised Older Adults: A Multicentre Observational Study of Prevalence, Associations and Outcomes. The journal of nutrition, health \& aging. 2017; 21: 830-6.

26 Kantoch A, Wielek J, Gryglewska B, Grodzicki T. Nutritional and Functional Status in Newly Hospitalized Older Patients Who are Not Underweight. Journal of nutrition in gerontology and geriatrics. 2017: 1-10.

27 Li XS, Obeid S, Klingenberg R, Gencer B, Mach F, Raber L, Windecker S, Rodondi N, Nanchen D, Muller O, Miranda MX, Matter CM, Wu Y, Li L, Wang Z, Alamri HS, Gogonea V, Chung YM, Tang WH, Hazen SL, et al. Gut microbiota-dependent trimethylamine N-oxide in acute coronary syndromes: a prognostic marker for incident cardiovascular events beyond traditional risk factors. European heart journal. 2017; 38: 814-24.

28 Kuhn T, Rohrmann S, Sookthai D, Johnson T, Katzke V, Kaaks R, von Eckardstein A, Muller D. Intra-individual variation of plasma trimethylamine-N-oxide (TMAO), betaine and choline over 1 year. Clinical chemistry and laboratory medicine : CCLM / FESCC. 2017; 55: 261-8.

29 Rohrmann S, Linseisen J, Allenspach M, von Eckardstein A, Muller D. Plasma Concentrations of Trimethylamine-N-oxide Are Directly Associated with Dairy Food Consumption and Low-Grade Inflammation in a German Adult Population. The Journal of nutrition. 2016; 146: 283-9. 


\section{Legends to Figures and Tables}

Figure 1. Kaplan-Meier estimates of recurrent VTE by TMAO levels. After 3 years, the cumulative incidence of recurrent VTE was higher in patients with medium and high levels of TMAO than in patients with low levels, but differences were not statistically significant (low, $11.0 \%$; medium, $15.8 \%$; high, $16.1 \% ; p=0.393)$.

Figure 2. Kaplan-Meier estimates to mortality by TMAO levels. After 3 years, the cumulative incidence of mortality varied significantly between patients with different levels of TMAO (low, $22.5 \%$; medium, $17.4 \%$; high, $27.7 \%$; $p$ = 0.030).

Figure 3. Relative hazards for mortality according to TMAO levels. Fractional polynomial Cox-regression showed a U-shaped association between log-transformed TMAO and death. The lowest risk of death was observed in patients presenting with TMAO around $4 \mu \mathrm{mol} / \mathrm{L}$.

Figure 4. Kaplan-Meier estimates of major or non-major bleeding by TMAO levels. After 3 years, the cumulative incidence of major or non-major bleeding was comparable between different levels of TMAO (low, $36.0 \%$; medium, $33.5 \%$; high, $36.3 \%, p=0.817$ ).

\section{Table 1. Baseline characteristics by TMAO levels.}

Values were missing in body mass index (1\%), systolic blood pressure (2\%), GFR (8\%), anaemia (6\%), and platelet count (6\%). Provoked VTE was defined as presence of $\geq 1$ of the following factors: major surgery, oestrogen therapy, or immobilization during the last 3 months; polypharmacy was defined as prescription of more than four different drugs. GFR = glomerular filtration rate, $\mathrm{IQ}=$ interquartile, $\mathrm{TMAO}=$ trimethylamine- $\mathrm{N}$-oxide; $\mathrm{PE}=$ pulmonary embolism, TIA = transient ischemic attack, VTE = venous thromboembolism . 


\section{Table 2. Association of TMAO with clinical end points after entire follow-up.}

VTE recurrence was adjusted for age, gender, overt PE, prior VTE, provoked index VTE and periods of anticoagulation. Mortality was adjusted for age, gender, overt PE, active cancer, immobilization during the last 3 months, chronic or acute heart failure, chronic lung disease and periods of anticoagulation. Bleeding was adjusted age, active cancer, history of major bleeding, antiplatelet therapy, overt PE, and periods of anticoagulation. $\mathrm{HR}=$ hazard ratio, IR = incidence rate (per 100 patient-years), $\mathrm{PE}=$ pulmonary embolism, SHR = subhazard ratio, TMAO = Trimethylamine- $\mathrm{N}$-oxide, $\mathrm{VTE}=$ venous thromboembolism .

\section{Table 3. Association of TMAO with Mortality - quadratic modeling.}

The adjusted joint $p$-value for both the linear and quadratic term was 0.045 . Mortality was adjusted for age, gender, overt PE, active cancer, immobilization during the last 3 months, chronic or acute heart failure, chronic lung disease and periods of anticoagulation. $\mathrm{HR}=$ hazard ratio, $\mathrm{IR}=$ incidence rate, $\mathrm{PE}=$ pulmonary embolism, $\mathrm{TMAO}=$ Trimethylamine- $\mathrm{N}-$ oxide, VTE = venous thromboembolism. 


\section{Tables}

Table 1. Baseline characteristics by TMAO levels.

\begin{tabular}{|c|c|c|c|c|c|}
\hline & All & $\begin{array}{l}\text { Low } \\
<2.28 \mu \mathrm{mol} / \mathrm{L}\end{array}$ & $\begin{array}{c}\text { Medium } \\
2.28-6.57 \mu \mathrm{mol} / \mathrm{L}\end{array}$ & $\begin{array}{l}\text { High } \\
>6.57 \mu \mathrm{mol} / \mathrm{L}\end{array}$ & $\begin{array}{c}\mathrm{p}- \\
\text { value }\end{array}$ \\
\hline & $\begin{array}{l}\text { n (\%) or median } \\
\text { (IQ-range) }\end{array}$ & $\begin{array}{l}\text { n (\%) or median } \\
\text { (IQ-range) }\end{array}$ & $\begin{array}{l}\text { n (\%) or median } \\
\text { (IQ-range) }\end{array}$ & $\begin{array}{l}\text { n (\%) or median } \\
\text { (IQ-range) }\end{array}$ & \\
\hline Total $\mathbf{N}$ & 859 & 214 & 430 & 215 & \\
\hline Age & $\begin{array}{c}75.0 \\
(69.0 ; 81.0)\end{array}$ & $\begin{array}{c}74.0 \\
(69.0 ; 80.0)\end{array}$ & $\begin{array}{c}74.0 \\
(69.0 ; 80.0)\end{array}$ & $\begin{array}{c}76.0 \\
(71.0 ; 83.0)\end{array}$ & 0.005 \\
\hline Female & $386(45 \%)$ & $104(49 \%)$ & $185(43 \%)$ & $97(45 \%)$ & 0.407 \\
\hline BMI & $\begin{array}{c}26.6 \\
(24.1 ; 29.8)\end{array}$ & $\begin{array}{c}26.2 \\
(23.8 ; 29.4)\end{array}$ & $\begin{array}{c}26.7 \\
(24.2 ; 29.8)\end{array}$ & $\begin{array}{c}27.0 \\
(23.8 ; 30.2)\end{array}$ & 0.222 \\
\hline Overt PE & $599(70 \%)$ & $164(77 \%)$ & $293(68 \%)$ & $142(66 \%)$ & 0.035 \\
\hline Prior VTE & $251(29 \%)$ & $54(25 \%)$ & $134(31 \%)$ & $63(29 \%)$ & 0.297 \\
\hline $\begin{array}{l}\text { Major surgery during the } \\
\text { last } 3 \text { months }\end{array}$ & $130(15 \%)$ & $40(19 \%)$ & $67(16 \%)$ & $23(11 \%)$ & 0.065 \\
\hline $\begin{array}{l}\text { Current oestrogen therapy } \\
\text { during the last } 3 \text { months }\end{array}$ & $27(3 \%)$ & $6(3 \%)$ & $14(3 \%)$ & $7(3 \%)$ & 0.951 \\
\hline $\begin{array}{l}\text { Immobilization during the } \\
\text { last } 3 \text { months }\end{array}$ & $185(22 \%)$ & $54(25 \%)$ & $95(22 \%)$ & $36(17 \%)$ & 0.094 \\
\hline Provoked index VTE & $252(29 \%)$ & $73(34 \%)$ & $132(31 \%)$ & $47(22 \%)$ & 0.014 \\
\hline Active cancer & $157(18 \%)$ & $43(20 \%)$ & $73(17 \%)$ & $41(19 \%)$ & 0.592 \\
\hline History of major bleeding & $89(10 \%)$ & $23(11 \%)$ & $38(9 \%)$ & $28(13 \%)$ & 0.257 \\
\hline Arterial hypertension & 549 (64\%) & $123(57 \%)$ & $271(63 \%)$ & $155(72 \%)$ & 0.006 \\
\hline Systolic blood pressure & $\begin{array}{c}135.0 \\
(120.0 ; 150.0)\end{array}$ & $\begin{array}{c}130.0 \\
(119.5 ; 148.5)\end{array}$ & $\begin{array}{c}138.0 \\
(125.0 ; 150.0)\end{array}$ & $\begin{array}{c}134.0 \\
(120.5 ; 146.5)\end{array}$ & 0.028 \\
\hline Chronic/acute heart failure & $102(12 \%)$ & $27(13 \%)$ & $47(11 \%)$ & $28(13 \%)$ & 0.687 \\
\hline Diabetes & $190(22 \%)$ & $43(20 \%)$ & $88(20 \%)$ & $59(27 \%)$ & 0.094 \\
\hline HbA1c [\%] & $5.7(5.4 ; 6.1)$ & $5.7(5.4 ; 6.1)$ & $5.7(5.4 ; 6.1)$ & $5.8(5.5 ; 6.2)$ & 0.088 \\
\hline $\begin{array}{l}\text { Cerebrovascular disease } \\
\text { (stroke, TIA) }\end{array}$ & $82(10 \%)$ & $16(7 \%)$ & $39(9 \%)$ & $27(13 \%)$ & 0.180 \\
\hline Chronic renal disease & $156(18 \%)$ & $24(11 \%)$ & $70(16 \%)$ & $62(29 \%)$ & $<0.001$ \\
\hline GFR $\left[\mathrm{mL} / \mathrm{min} / 1.73 \mathrm{~m}^{2}\right]$ & $\begin{array}{c}58.1 \\
(47.4 ; 73.6)\end{array}$ & $\begin{array}{c}66.2 \\
(53.6 ; 82.2)\end{array}$ & $\begin{array}{c}58.5 \\
(49.3 ; 74.1)\end{array}$ & $\begin{array}{c}49.6 \\
(36.7 ; 62.0)\end{array}$ & $<0.001$ \\
\hline Anaemia & $333(39 \%)$ & $94(44 \%)$ & $156(36 \%)$ & $83(39 \%)$ & 0.378 \\
\hline Platelet count $<150 \mathrm{G} / \mathrm{l}$ & $130(15 \%)$ & $25(12 \%)$ & $69(16 \%)$ & $36(17 \%)$ & 0.171 \\
\hline Polypharmacy & 439 (51\%) & $108(50 \%)$ & $214(50 \%)$ & $117(54 \%)$ & 0.525 \\
\hline $\begin{array}{l}\text { Anticoagulation prior to } \\
\text { index VTE }\end{array}$ & $41(5 \%)$ & $13(6 \%)$ & $20(5 \%)$ & $8(4 \%)$ & 0.513 \\
\hline $\begin{array}{l}\text { Type of initial parenteral } \\
\text { anticoagulation }\end{array}$ & & & & & 0.094 \\
\hline LMWH & $410(48 \%)$ & $97(45 \%)$ & $212(49 \%)$ & $101(47 \%)$ & \\
\hline UFH & $272(32 \%)$ & $79(37 \%)$ & $123(29 \%)$ & $70(33 \%)$ & \\
\hline Fondaparinux & $147(17 \%)$ & $27(13 \%)$ & $81(19 \%)$ & $39(18 \%)$ & \\
\hline Danaparoid & $1(0 \%)$ & $0(0 \%)$ & $0(0 \%)$ & $1(0 \%)$ & \\
\hline
\end{tabular}




\begin{tabular}{|l|c|c|c|c|c|}
\hline None & $29(3 \%)$ & $11(5 \%)$ & $14(3 \%)$ & $4(2 \%)$ & \\
\hline Initial VKA therapy & $746(87 \%)$ & $178(83 \%)$ & $380(88 \%)$ & $188(87 \%)$ & 0.177 \\
\hline Antiplatelet therapy & $275(32 \%)$ & $62(29 \%)$ & $138(32 \%)$ & $75(35 \%)$ & 0.422 \\
\hline Aspirin & $243(28 \%)$ & $57(27 \%)$ & $120(28 \%)$ & $66(31 \%)$ & 0.627 \\
\hline
\end{tabular}


Table 2. Association of TMAO with clinical end points after entire follow-up.

\begin{tabular}{|c|c|c|c|c|c|c|c|c|}
\hline End point & $\begin{array}{l}\text { TMAO } \\
\text { levels }\end{array}$ & $\begin{array}{c}\mathrm{N}^{\circ} \text { of } \\
\text { patients }\end{array}$ & $\begin{array}{c}\mathrm{N}^{\circ} \text { events/ } \\
\text { patient- } \\
\text { years }\end{array}$ & IR (95\% Cl) & $\begin{array}{l}\text { Crude SHR or } \\
\text { HR }(95 \% \mathrm{Cl})\end{array}$ & $\begin{array}{c}\text { p- } \\
\text { value }\end{array}$ & $\begin{array}{l}\text { Adjusted SHR or } \\
\text { HR }(95 \%-\mathrm{Cl})\end{array}$ & $\begin{array}{c}p- \\
\text { value }\end{array}$ \\
\hline \multirow{4}{*}{$\begin{array}{l}\text { VTE } \\
\text { recurrence }\end{array}$} & All & 859 & 106 / 1809.1 & 5.9 (4.8 to 7.1$)$ & & & & \\
\hline & Low & 214 & $19 / 446.6$ & 4.3 (2.7 to 6.7 ) & 1 (reference) & & 1 (reference) & \\
\hline & Medium & 430 & $57 / 924.3$ & 6.2 (4.8 to 8.0 ) & 1.48 (0.88 to 2.49$)$ & 0.142 & 1.38 (0.81 to 2.36$)$ & 0.232 \\
\hline & High & 215 & $30 / 438.2$ & 6.8 (4.8 to 9.8 ) & 1.56 (0.87 to 2.77$)$ & 0.133 & 1.44 (0.80 to 2.58$)$ & 0.221 \\
\hline \multirow[t]{4}{*}{ Mortality } & All & 859 & 179 / 1921.2 & 9.3 (8.0 to 10.8 ) & & & & \\
\hline & Low & 214 & $46 / 456.0$ & 10.1 (7.6 to 13.5$)$ & 1 (reference) & & 1 (reference & \\
\hline & Medium & 430 & 75 / 1003.4 & 7.5 (6.0 to 9.4$)$ & 0.75 (0.52 to 1.09$)$ & 0.130 & $0.68(0.47$ to 0.98$)$ & 0.039 \\
\hline & High & 215 & $58 / 461.7$ & 12.6 (9.7 to 16.2$)$ & 1.21 (0.82 to 1.78$)$ & 0.340 & 1.02 (0.68 to 1.52$)$ & 0.922 \\
\hline \multirow{4}{*}{$\begin{array}{l}\text { Major or } \\
\text { non-major } \\
\text { bleeding }\end{array}$} & All & 859 & 259 / 1565.1 & 16.5 (14.7 to 18.7$)$ & & & & \\
\hline & Low & 214 & $66 / 374.2$ & 17.6 (13.9 to 22.4$)$ & 1 (reference) & & 1 (reference) & \\
\hline & Medium & 430 & $126 / 799.2$ & 15.8 (13.2 to 18.8$)$ & 0.95 (0.71 to 1.28$)$ & 0.753 & $0.96(0.72$ to 1.28$)$ & 0.775 \\
\hline & High & 215 & $67 / 391.7$ & 17.1 (13.5 to 21.7$)$ & $1.01(0.72$ to 1.42$)$ & 0.933 & 0.94 (0.67 to 1.32$)$ & 0.732 \\
\hline
\end{tabular}


Table 3. Association of TMAO with Mortality - quadratic modeling.

\begin{tabular}{|l|c|c|c|c|c|}
\hline End point & TMAO levels & $\begin{array}{c}\text { Crude HR } \\
\mathbf{( 9 5 \% ~ C l )}\end{array}$ & $\begin{array}{c}\mathbf{p}- \\
\text { value }\end{array}$ & $\begin{array}{c}\text { Adjusted HR } \\
\mathbf{( 9 5 \% - C l )}\end{array}$ & $\begin{array}{c}\mathbf{p}- \\
\text { value }\end{array}$ \\
\hline \multirow{2}{*}{ Mortality } & $\log (\mathrm{TMAO})$ & $0.73(0.53$ to 1.00$)$ & 0.048 & $0.73(0.53$ to 0.98$)$ & 0.039 \\
\cline { 2 - 6 } & $\log (\mathrm{TMAO})^{2}$ & $1.13(1.04$ to 1.23$)$ & 0.004 & $1.11(1.02$ to 1.21$)$ & 0.013 \\
\hline
\end{tabular}




\section{Figures}

Figure 1
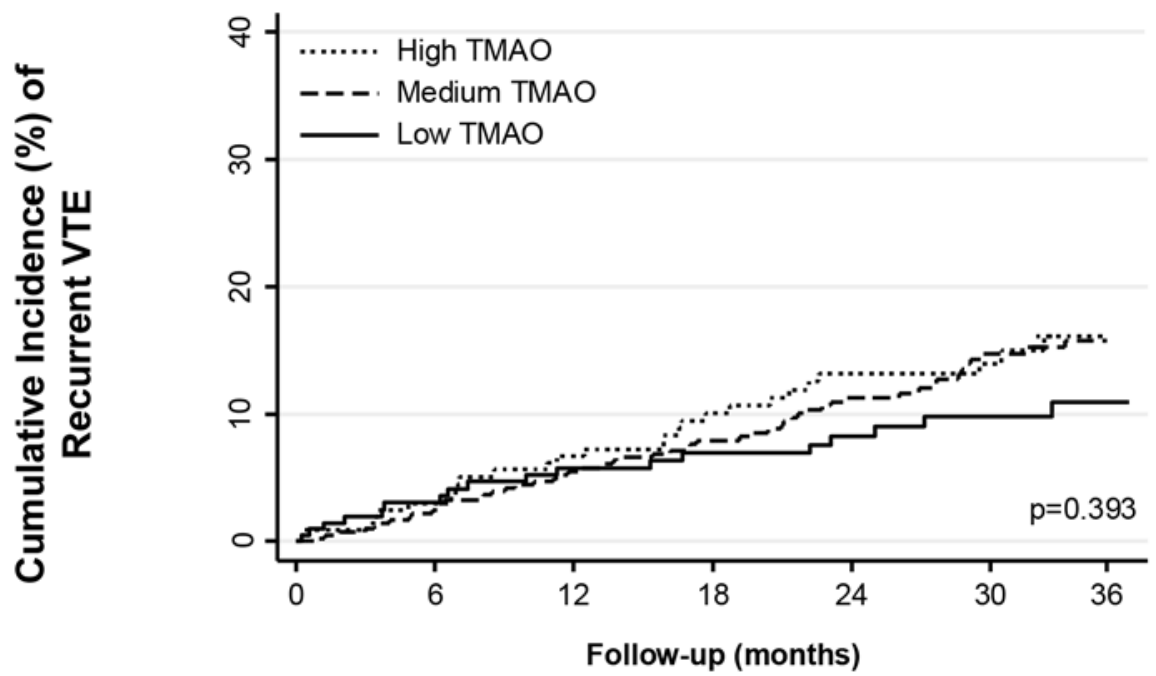

Number at risk

High TMAO

Medium TMAO

$\begin{array}{ll}215 & 183 \\ 430 & 386\end{array}$

$83 \quad 171$

$171 \quad 147$

323

125

$214 \quad 177$

164

151

249

$88 \quad 51$

LOW TMAO

\section{Figure 2}
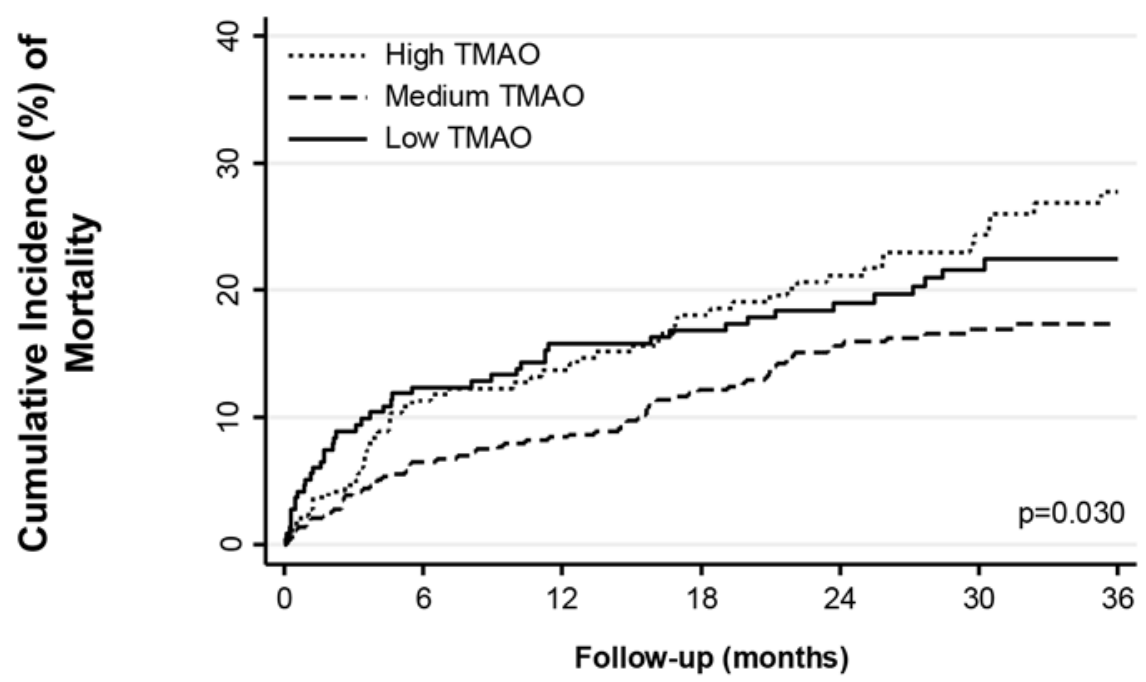

Number at risk

$\begin{array}{llllllcc}\text { High TMAO } & 215 & 186 & 180 & 160 & 139 & 98 & 61 \\ \text { Medium TMAO } & 430 & 392 & 378 & 347 & 275 & 211 & 135 \\ \text { Low TMAO } & 214 & 179 & 169 & 158 & 129 & 95 & 61\end{array}$




\section{Figure 3}
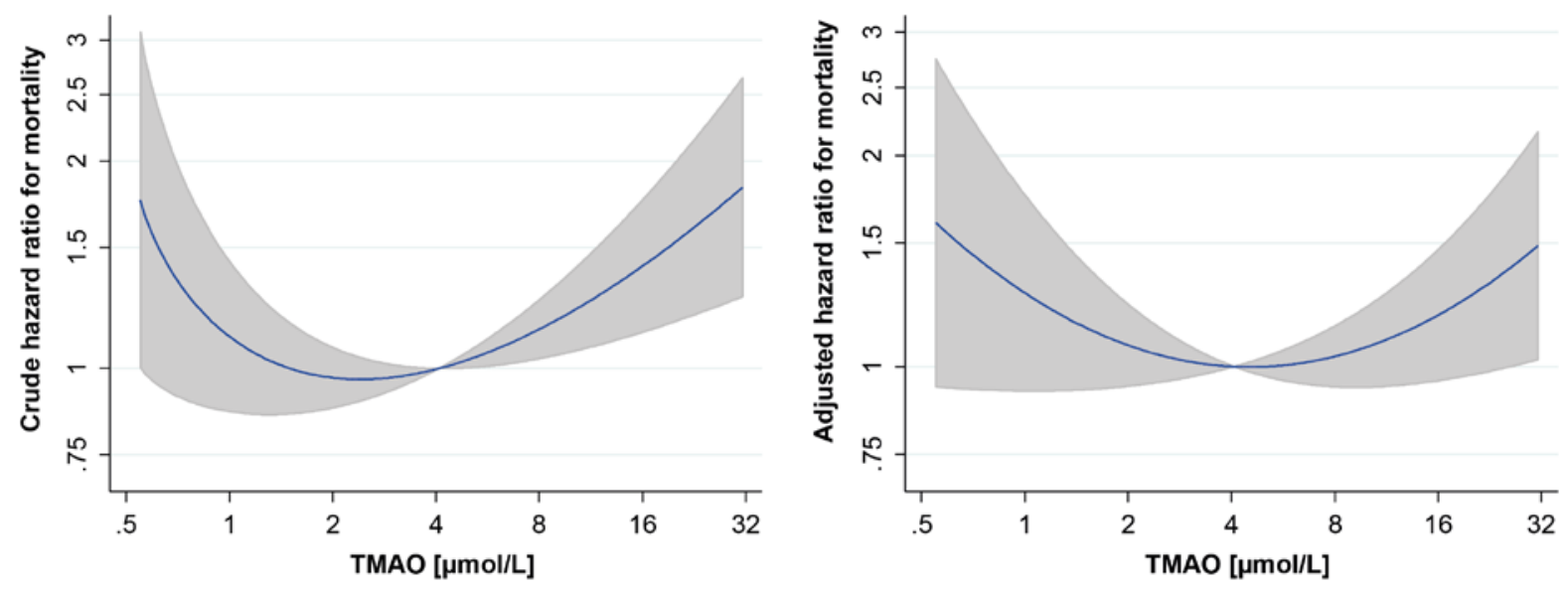

\section{Figure 4}
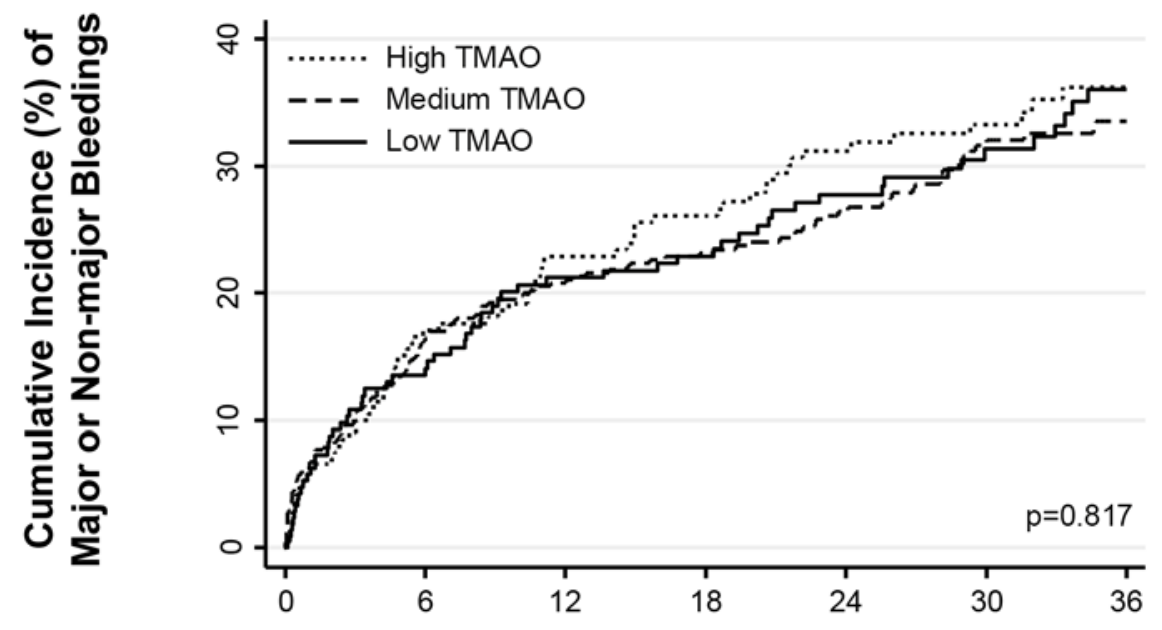

Follow-up (months)

Number at risk

$\begin{array}{llllllll}\text { High TMAO } & 215 & 161 & 146 & 132 & 110 & 74 & 44 \\ \text { Medium TMAO } & 430 & 336 & 308 & 279 & 214 & 155 & 97 \\ \text { Low TMAO } & 214 & 157 & 140 & 132 & 109 & 78 & 46\end{array}$




\section{Supplemental Table}

Supplemental Table 1. Subgroup analysis for mortality.

\begin{tabular}{|c|c|c|c|c|c|c|}
\hline Subgroup & $\begin{array}{l}\text { TMAO } \\
\text { levels }\end{array}$ & $\begin{array}{l}N^{\circ} \text { events } / \\
N^{\circ} \text { patients }\end{array}$ & $\begin{array}{c}\text { Crude HR } \\
(95 \% \mathrm{Cl})\end{array}$ & $\begin{array}{c}\text { p- } \\
\text { value }\end{array}$ & $\begin{array}{l}\text { Adjusted HR } \\
(95 \%-\mathrm{Cl})\end{array}$ & p-value \\
\hline \multirow{4}{*}{$\begin{array}{l}\text { Patients with } \\
\text { overt PE }\end{array}$} & All & $121 / 599$ & & & & \\
\hline & Low & $37 / 164$ & 1 (reference) & & 1 (reference) & \\
\hline & Medium & $49 / 293$ & 0.70 (0.46 to 1.07 ) & 0.101 & 0.65 (0.42 to 0.99 ) & 0.047 \\
\hline & High & $35 / 142$ & 1.04 (0.66 to 1.66 ) & 0.854 & 0.94 (0.58 to 1.51 ) & 0.788 \\
\hline \multirow{4}{*}{$\begin{array}{l}\text { Patients with } \\
\text { DVT only }\end{array}$} & All & $58 / 260$ & & & & \\
\hline & Low & $9 / 50$ & 1 (reference) & & 1 (reference & \\
\hline & Medium & $26 / 137$ & 0.95 (0.44 to 2.02 ) & 0.887 & 0.92 (0.41 to 2.04 ) & 0.829 \\
\hline & High & $23 / 73$ & 1.74 (0.80 to 3.76 ) & 0.160 & 1.53 (0.67 to 3.49 ) & 0.308 \\
\hline \multirow{4}{*}{$\begin{array}{l}\text { Patients } \\
\text { without } \\
\text { cancer }\end{array}$} & All & $96 / 702$ & & & & \\
\hline & Low & $22 / 171$ & 1 (reference) & & 1 (reference & \\
\hline & Medium & $41 / 357$ & 0.86 (0.51 to 1.45$)$ & 0.573 & 0.99 (0.59 to 1.68$)$ & 0.979 \\
\hline & High & $33 / 174$ & 1.46 (0.85 to 2.50 ) & 0.172 & 1.37 (0.79 to 2.36 ) & 0.266 \\
\hline \multirow{4}{*}{$\begin{array}{l}\text { Patients with } \\
\text { cancer }\end{array}$} & All & $83 / 157$ & & & & \\
\hline & Low & $24 / 43$ & 1 (reference) & & 1 (reference & \\
\hline & Medium & $34 / 73$ & 0.62 (0.37 to 1.05 ) & 0.077 & 0.55 (0.32 to 0.95$)$ & 0.032 \\
\hline & High & $25 / 41$ & 0.89 (0.51 to 1.57$)$ & 0.694 & 0.71 (0.39 to 1.30$)$ & 0.267 \\
\hline
\end{tabular}

The adjusted HR in subgroup analysis for patients with overt PE and DVT only was corrected for age, gender, active cancer, immobilization during the last 3 months, chronic or acute heart failure, chronic lung disease and $A C$ as time-varying covariate. The adjusted HR in subgroup analysis for patients with vs. without cancer was corrected for age, gender, overt $\mathrm{PE}$, immobilization during the last 3 months, chronic or acute heart failure, chronic lung disease and $\mathrm{AC}$ as time-varying covariate. $\mathrm{HR}=$ hazard ratio, DVT $=$ deep venous thrombosis, $\mathrm{PE}=$ pulmonary embolism, TMAO = Trimethylamine- $\mathrm{N}$-oxide. 